ANUARIO DE Estudios MEDIEVALES

49/1, enero-junio de 2019, pp. 75-101

ISSN 0066-5061

https://doi.org/10.3989/aem.2019.49.1.03

\title{
CATECHESI E DRAMMATIZZAZIONE: LO SPIRITO SANTO NEI SERMONI DI PENTECOSTE DI VICENT FERRER
}

\author{
CATECHESIS AND DRAMATISATION: \\ THE HOLY SPIRIT IN VINCENT FERRER'S PENTECOST SERMONS
}

PIETRO DELCORNO

University of Leeds / University of the Witwatersrand http://orcid.org/ 0000-0002-1556-3215

\begin{abstract}
L'articolo si concentra sull'abilità comunicativa di Vicent Ferrer e sulla sua capacità di miscelare catechesi e invenzione narrativa, analizzando sei versioni del sermone Repleti sunt omnes Spiritu Sancto. Gli schemi, le reportationes, i sermoni modello delle prediche tenute da Ferrer in diverse parti d'Europa mostrano come la festa di Pentecoste fornisse l'occasione per una vivace 'messa in scena' del racconto biblico e come la predicazione fornisse agli ascoltatori un accesso, mediato ma non banale, alla conoscenza della Scrittura. Nel sermone di Pentecoste, indicando la comunità apostolica come modello per i suoi ascoltatori, Ferrer sviluppava una riflessione tanto sul ruolo della predicazione quanto sul disporsi ad accogliere lo Spirito Santo.
\end{abstract}

Parole chiave: predicazione; Vicent Ferrer; Spirito Santo; Bibbia; manoscritti.

Abstract: The article focuses on the communicative skills of Vincent Ferrer and on his ability to merge catechesis and narrative inventiveness. The analysis is conducted on six versions of the sermon Repleti sunt omnes Spiritu Sancto (notes, reportationes, and model sermons), which were preached by Ferrer in different parts of Europe. The texts highlight how Pentecost provided Ferrer with the opportunity to develop a lively dramatisation of the biblical episode and how preaching gave to the faithful a mediated, and yet not simplistic access to the Bible. By presenting the apostolic community as the exemplary model for his listeners, Ferrer elaborated on both the role of preaching and the way to welcome the Holy Spirit. Keywords: preaching; Vincent Ferrer; Holy Spirit; Bible; manuscripts.

\section{SOMMARIO}

1. Introduzione.- 2. Lo schema del manoscritto di Perugia.- 3. La predica di La Moraleja.- 4. La reportatio di Tortosa.- 5. La reportatio del 1416.-6. Il testo standardizzato: drammatizzare la Pentecoste.- 7. Conclusione.- 8. Bibliografia citata.

\section{INTRODUZIONE ${ }^{1}$}

Nel sistema di comunicazione tardomedievale, la predicazione aveva il compito non solo di mediare contenuti dottrinali o morali, ma

\footnotetext{
${ }^{1}$ Abbreviazioni usate: $E=$ Vicent Ferrer, Sermones de tempore, 3 voll., Colonia, Heinrich Quentell, vol. I.; $P=$ Vicent Ferrer, Sermones, Perugia, Convento dei Domenicani, MS 477; $T=$ Vicent Ferrer, Sermones de tempore, Toulouse, Bibliothèque municipale, MS 345.

Citation / Cómo citar este artículo: Delcorno, Pietro (2019), Catechesi e drammatizzazione: Lo Spirito Santo nei sermoni di Pentecoste di Vicent Ferrer, "Anuario de Estudios Medievales" 49/1, pp. 75-101. https://doi.org/10.3989/aem.2019.49.1 .03

Copyright: (C) 2019 CSIC. This is an open-access article distributed under the terms of the Creative Commons Attribution 4.0 International (CC BY 4.0) License.
} 
anche di convincere e spingere all'azione gli ascoltatori ${ }^{2}$. Stando alle parole dei predicatori del tempo, si trattava di accendere un fuoco, trasmettendo l'energia trasformante dello Spirito ${ }^{3}$. Per spiegarlo, Vicent Ferrer $(\dagger 1419)^{4}$ utilizza l'immagine della pietra focaia capace di sprigionare una scintilla e appiccare fuoco a un'esca: la pietra è Cristo, il ferro è la lingua del predicatore, il luogo dove si pone l'esca è la piazza, mentre l'esca sono i peccati degli ascoltatori. La parola, anzi, la performance del predicatore è una scintilla che fa conoscere i peccati e maturare nuove intenzioni, spingendo così al cambiamento ${ }^{5}$.

L'affermazione è contenuta in un sermone per Pentecoste che ha per thema il versetto Repleti sunt omnes Spiritu Sancto (Atti 2,4) e che, nelle sue diverse forme, sarà al centro di questo articolo ${ }^{6}$. In questo sermone, Ferrer insegnava agli ascoltatori come disporsi a ricevere lo Spirito, proponendo loro come modello la comunità apostolica. Introdurre una riflessione sulla potenza della predicazione (l'immagine della pietra focaia) in un sermone di Pentecoste non era inusuale, in quanto la festa -centrale nel calendario liturgico- costituiva il momento fondante della predicazione cristiana, iniziata dagli apostoli ricolmi della forza e dell'eloquenza concessa loro dallo Spirito. Pentecoste costituiva così une sorte de mythe de fondation de la fonction même du prédicateur ${ }^{7}$. Come osserva Silvana Vecchio riguardo ai sermoni duecenteschi, la festa forniva una sorta di autolegittimazione dei predicatori che spesso usavano i sermoni di quel giorno pour se mettre euxmêmes en scène, parler de leur métier, illustrer les fondements théologiques de leur dignité, et revendiquer la supériorité de leur parole sur toute autre parole $^{8}$. I sermoni del Quattrocento sembrano insistere meno su questo tema,

\footnotetext{
${ }^{2}$ Si veda Muzzarelli 2014. Per un'introduzione alla predicazione medievale: Kienzle 2000; Muessig 2002; Bériou 2018. Un'aggiornata bibliografia in Delcorno 2017b.

${ }^{3}$ Muessig 2015; Delcorno 2017a.

${ }^{4}$ Per orientarsi nella bibliografia dedicata alla predicazione di Ferrer: Cátedra 1994; Perarnau 1999a; Martínez Romero 2002; Callado Estela 2007. Riguardo alla predicazione iberica: Sánchez 2000, 2011; Catalán 2013.

5 "Si yo tenia ací hun foguer e volia encendre foch, veus que pendria la pedra e-l esclavó, e axí ferint de la pedra en l'esclavó, devallaria lo foch en la escha. Sus axí és: la pedra és Jesuchrist (...), lo ferret és la lengua del preycador; lo esquer on està la esqua, és la plaça de la preycació; la escha, que és freda e negra, açò són los peccats negres, e teniu-los vosaltres. Quants peccats hi ha ací ara. E quan lo preycador preyca, veus que cau una purna de foch, e encén-se en tu, e coneixs lo teu peccat, e prens ferm propòsit de humiliar-te e de restituir los torts, e la manceba, etc. Vet, lo sant Spirit és vengut en tu"; Ferrer, Sermons, ed. Sanchis 19321934, vol. I, p. 142. Su questo tema: Esponera 2007. Sul fuoco come attributo iconografico di Ferrer: Rusconi 2008.

${ }^{6}$ Schema 747 in Perarnau 1999d.

${ }^{7}$ Vecchio 2008, p. 244. Si veda anche Cooper-Rompato 2016.

${ }^{8}$ Vecchio 1997, p. 256. Si veda anche Delmas 2019.
} 
privilegiando un discorso più ampio sul modo per prepararsi ad accogliere lo Spirito e sui suoi effetti nella vita dei fedeli ${ }^{9}$. I temi potevano in realtà intrecciarsi, come appare nel sermone di Ferrer, dove l'esaltazione della predicazione e la catechesi ai fedeli su come accogliere lo Spirito procedono di pari passo.

Inoltre, il sermone qui analizzato mostra in maniera esemplare l'abilità di Ferrer nel presentare in forme semi-drammatiche e con finalità catechetiche un racconto biblico, quale quello di Pentecoste. Lo studio di questo testo contribuisce a mettere in luce le vie attraverso le quali la predicazione permetteva ai fedeli un accesso, in forme mediate e controllate, ma non banali, alla conoscenza della Scrittura ${ }^{10}$. Gli stessi predicatori non mancano di sottolineare i limiti di questa conoscenza per i laici, i quali non possono attingere direttamente alla Bibbia. In una predica del 1416, Ferrer presenta Cristo come il ricco del racconto evangelico, intento a imbandire un lauto banchetto dal quale cadono preziose briciole (Luca 16,21). In quello che risulta un condensato della propria predicazione, Ferrer descrive quattro tavole imbandite da Dio: la mensa della penitenza, quella eucaristica, quella sapienziale $\mathrm{e}$, infine, il banchetto celeste ${ }^{11}$. La terza mensa è quella della Scrittura, accostabile direttamente solo da teologi e chierici, capaci di interpretare il significato anagogico, allegorico e tropologico di quanto leggono, là dove i laici -come Lazzaro nel Vangelo- hanno accesso solo alle briciole $^{12}$. Nel sermone, queste ultime rappresentano il cibo necessario per la vita cristiana dei fedeli e sono identificate con i più diffusi schemi catechetici: il Credo, il Decalogo, il Pater noster, la prassi penitenziale. Il concetto è ribadito in un altro sermone, strutturato intorno al tema dei quattro libri disposti da Dio per istruire l'uomo, dove Ferrer sottolinea come la Bibbia sia uno libro spirituale, quia non omnes sciunt in illo legere, nisi litterati et clerici $^{13}$. Citando una visione di Ezechiele, si dice che questo libro è scriptus intus et foris: dentro per i chierici che ne intendono il significato spirituale, mentre fuori vi sono i segreti estratti dagli apostoli pro rudibus et simplicibus, ovvero gli articoli del Credo, dove si riassume quanto tutti devono sapere e credere $^{14}$.

\footnotetext{
${ }^{9}$ Delcorno 2019.

${ }^{10}$ Per una prima introduzione: Delcorno 1996; Wenzel 2012.

${ }^{11}$ Ferrer, Sermons, ed. Sanchis 1932-1934 vol. II, p. 24. Su questo sermone Delcorno 2011.

${ }^{12}$ Ferrer, Sermons, ed. Sanchis 1932-1934 vol. II, p. 29. Una lettura positiva della mensa dell'epulone è rara nell'esegesi medievale: Delcorno 2014a, pp. 30-34. Sulla polisemia dell'immagine delle briciole, Maldina 2016.

${ }^{13}$ Perarnau 2011-2013, p. 546. I quattro libri sono la natura, la Scrittura, la grazia (il libro che si legge guardando Cristo crocifisso) e la coscienza.

${ }^{14}$ Ibidem, p. 547.
} 
In realtà, l'accesso alla Scrittura offerto ai laici non si riduceva a questi nudi schemi catechetici. La predicazione stessa svolgeva una ben più ampia e capillare opera di mediazione, servendosi anche di vivaci e fantasiose "messe in scena" dei racconti biblici. Proprio la capacità di attualizzare o reinventare in forme semi-drammatiche la Scrittura dal pulpito rappresenta uno degli aspetti centrali della cosiddetta 'pratica della Bibbia' nella società tardomedievale ${ }^{15}$. I sermoni di Ferrer sono un precoce esempio delle forme più creative e spregiudicate di drammatizzare la Bibbia attraverso racconti emotivamente avvincenti e ricchi di dettagli, personaggi, gesti, dialoghi presentati attraverso l'abile performance del predicatore ${ }^{16}$. Ferrer è tra i primi a sviluppare appieno uno degli aspetti più significativi della comunicazione religiosa del Quattrocento, visibile tanto in predicatori famosi, come Bernardino da Siena e Michel Menot, quanto in protagonisti meno noti, come l'anonimo autore del Quadragesimale peregrini e Johannes Meder ${ }^{17}$.

Infine, studiando le varie attestazioni del sermone Repleti sunt omnes attraverso l'eccezionale corpus di testi di Ferrer, sarà possibile cogliere sia come il domenicano di Valencia riutilizzasse e variasse i propri schemi lungo il suo itinerario di predicazione europeo, sia come i suoi sermoni vennero trasmessi alle future generazioni di predicatori, contribuendo così a indagare la complessa relazione tra reportationes connesse -almeno in origine- a una specifica performance orale e l'elaborazione dei successivi sermoni modello ${ }^{18}$.

Seguendo un ordine cronologico, studieremo questo sermone in sei versioni giunte fino a noi: lo schema contenuto nel sermonario di Perugia (1407); la reportatio della predica del 1411 a La Moraleja; il sermone predicato a Tortosa, verosimilmente nel 1413; la reportatio di un sermone predicato probabilmente ad Albi nel 1416; il sermone modello latino (con inserti in volgare) attestato in un manoscritto della seconda metà del Quattrocento e, infine, la prima edizione a stampa (1485) che fissò il testo per i secoli seguenti.

\footnotetext{
${ }^{15}$ Boynton, Reilly 2011; Delcorno 2017a.

${ }^{16}$ Si veda Martínez Romero 2002, pp. 151-171. Per una comparazione del modo di drammatizare il racconto biblico in Ferrer, Bernardino da Siena e nel teatro confraternale: Delcorno 2014b. Sul rapporto tra predicazione e performance Bouhaïk-Gironès, Polo de Beaulieu 2013.

${ }^{17}$ Delcorno 2011; 2017a, pp. 192-212 (Bernardino), pp. 310-354 (Meder), pp. 372-377 (Menot). Il Quadragesimale peregrini, opera di un francescano attivo negli stessi anni di Ferrer, si basa su una riscrittura dell'itinerario dantesco nell'aldilà; Delcorno $2017 \mathrm{c}$.

${ }^{18}$ Le reportationes permettono di indagare "the stimulating tension posed by the gap between the written accounts that remain and the medeival experiences of what was fundamentally an oral and performative genre"; Thayer 2014, p. 12. Riguardo a Ferrer: Cátedra 1994, pp. 83-99; Cabré, Renedo 1996; Renedo 2007; Losada 2015. Un confronto tra reportationes e sermoni modello in Perarnau 1985, pp. 327-361.
} 


\section{LO SCHEMA DEL MANOSCRITTO DI PERUGIA}

La prima attestazione del sermone Repleti sunt omnes è lo schema 192 nel manoscritto 477 conservato presso il Convento dei Domenicani di Perugia. Il manoscritto raccoglie i sermoni utilizzati da Ferrer durante la sua predicazione in Italia nel $1407-1408^{19}$. Si tratta di un testo di lavoro per predicatori, composto di stringate note in latino che, in poche linee, forniscono l'ossatura di un discorso. Stava al futuro lettore interpretarle e svilupparle in un sermone vero e proprio. Riporto l'intero schema ${ }^{20}$.

[f. 37r] Dominica pentecostes.

Repleti sunt omnes spiritu sancto. Ac 2 [2,4]. Nota quatuor dispositiones ad recipiendum spiritum, scilicet:

$<$ Abs $>$ tinentia corporalis.

Oratio affectualis.

Concordia fraternalis.

Audientia doctrinalis.

Omnes iste fuerunt plene in discipulis Yesu, secundo: Repleti etc.

De prima. Siccatio lignorum precedit ignitionem. Ez $37[37,4]$ :

Ossa arida audite etc. [verbum Domini, hec dicit Dominus Deus ossibus his: Ecce ego intromittam in vos spiritum et vivetis]. Hec fuit in Christi dicipulis. Mr 2 [2,20]: Venient dies cum auferetur ab eis sponsus etc. [et tunc ieiunabunt in illa die]. De patris ergo lumine decorus ignis etc. [advenit I qui fida Christi pectora I calore verbi compleat $]^{21}$.

De secunda. Emisso ab ore anelitu calido, atraheretur aura recens. Ps 117 [118,131]: Os meum aperui etc. [et adtraxi spiritum]. Hec fuit in eis: Hii omnes erant perseverantes etc. [unanimiter in oratione] Ac $1[1,14]$. Dum hora cunctis tertia ect. [repente mundus intonat, I apostolis orantibus I Deum venisset nuntians] $]^{22}$.

[f. 37v] De tertia. Coniuctio membrorum requiritur ad inhabitationem spiritus vitalis. Iude unico [1,19]: Hii qui segregant semetipsos, animales spiritum non habentes. Hec fuit in discipulis, Ac 2 [2,1]: Dum complerentur dies Pentechostes etc. [erant omnes

\footnotetext{
${ }^{19}$ Il manoscritto contiene 477 schemi, editi in Ferrer, Sermonario de Perugia, ed. Gimeno, Mandingorra 2006. Su questo e altri due manoscritti collegati alla medesima tradizione (Biblioteca Apostolica Vaticana, MS Vat. lat. 4375 e MS Vat. lat 7730): Perarnau 1999b. I tre manoscritti contengono tutti lo schema di Pentecoste. Sulla predicazione di Ferrer in Italia: Gaffuri 2006.

${ }^{20}$ P, f. 37r-v. Mi avvalgo del manoscritto rispetto a Ferrer, Sermonario de Perugia, ed. Gimeno, Mandingorra 2006, p. 278. Nella trascrizione sciolgo le abbreviazioni, adattato la punteggiatura e le maiuscole secondo l'uso moderno, completo i riferimenti biblici e liturgici per renderli intellegibili. Conservo le sottolineature presenti nel manoscritto.

${ }^{21}$ Inno Iam Christus astra ascenderat; Walsh, Husch 2012, pp. 184-187.

${ }^{22}$ Ibidem.
} 
pariter in eodem loco]. Invenit eos concordes caritate etc. [et illustravit eos inundans divinitas deitatis ${ }^{23}$.

De quarta. Percussio lapidis ad ferrum excutit ignem super escam. Ac $10[10,44]$ : Ad huc loquente Petro verba hec etc. [cecidit spiritus sanctus super omnes]. Hec fuit in discipulis audientibus doctrinam virginis. Sap 8 [8,4]: Doctrix est discipline Dei etc. [et electrix operum illius]. Nota virginis doctrinam, ad discipulos mirantes de dilatione promissionis Christi, iuxta illud Patrata sunt hec mistice etc. [pasche peracto tempore I sacro dierum numero I quo lege fit remissio] $]^{24}$.

Lo schema elenca quattro dispositiones necessarie per ricevere lo Spirito e invita gli ascoltatori a guardare all'esempio della comunità apostolica riunita nel cenacolo di Gerusalemme. In forma condensata fino ai limiti dell'intelligibilità, per ciascuna disposizione si annotano: un'immagine tratta dal mondo naturale e interpretata attraverso un versetto biblico; l'esemplare comportamento dei discepoli in attesa dello Spirito, con un opportuno riferimento biblico; il rimando a uno degli inni liturgici di Pentecoste.

Ad esempio, riguardo alla abstinentia corporalis si scrive: "De 1a. Siccatio lignorum precedit ignitionem; Ez 37: Ossa arida audite etc. Hec fuit in Christi dicipulis; Mr 2: Venient dies cum auferetur ab eis sponsus etc. De patris ergo lumine d. $i$. etc.". Come si deve seccare la legna prima che possa prendere fuoco, così l'astinenza e il digiuno servono per prepararsi a ricevere lo Spirito. Il nesso tra "secchezza" del corpo e dono dello Spirito è illustrato attraverso la visione delle ossa aride in Ezechiele 37, dando per scontato che il lettore comprenda e completi il versetto incominciato. Per dimostrare che i discepoli si prepararono digiunando, si richiama Marco 2,20 dove Gesù affermava che i suoi discepoli avrebbero digiunato quando lo sposo sarebbe stato loro tolto, un annuncio applicato ai giorni tra l'ascensione di Cristo e la discesa dello Spirito. Infine, si annota un passo dell'inno Iam Christus, dove è ripreso il tema del fuoco dello Spirito che riempie il cuore dei fedeli con il calore della parola divina, o meglio del Verbum, come afferma la diffusissima Expositio hymnorum, in cui si dice anche che tale dono concede agli apostoli e ai fedeli la scientia loquendi ${ }^{25}$.

\footnotetext{
${ }^{23}$ Responsorio di Pentecoste: Advenit ignis; Hesbert 1963-1979, n. 6053.

${ }^{24}$ Inno Beata nobis gaudia; Walsh, Husch 2012, pp. 188-191.

25 "ut haberent scientiam loquendi pectora apostolorum vel hominum fida, id est fideli a Christi, et hoc cum calore, id est ardore spiritussancti, verbi, id est filii"; Expositio 1488, f. 51r. Su questa raccolta, databile al XII secolo, Moss 1987, pp. 115-118, dove si ricorda come alcune edizioni a stampa "advertise its usefulness to preachers wishing to use the appropriate hymns as a source for sermons for saints' days" (p. 118). La conoscenza degli inni liturgici e della loro interpretazione faceva parte della formazione di base, tanto che nelle biblioteche dei conventi l'Expositio era spesso collocata tra le grammatiche; Gneuss 1968, pp. 194-198.
} 
Il riferimento sistematico agli inni liturgici è un tratto specifico di questo schema. In particolare, presentando la quarta dispositio, un inno liturgico riassume il contenuto dell'insegnamento impartito dalla Vergine agli apostoli, perplessi dall'apparente ritardo dello Spirito. L'ascolto della doctrina è infatti indicato come l'elemento decisivo per far divampare il fuoco dello Spirito, come testimonia l'episodio della discesa dello Spirito su Cornelio e la sua famiglia, avvenuta mentre l'apostolo Pietro stava predicando (Atti 10,44). Parlando dei discepoli che si erano preparati a Pentecoste ascoltando la Vergine, identificata con la doctrix discipline Dei (Sapienza 8,4), il contenuto della sua 'predicazione' è indicato attraverso un passo di un altro inno, Beata nobis gaudia, dove si sottolinea il significato simbolico dei 50 giorni tra Pasqua e Pentecoste, collegandolo sia al tempo intercorso tra l'uscita dall'Egitto e il dono della legge sul Sinai, sia alla liberazione dalla schiavitù nell'anno giubilare ${ }^{26}$.

Nel comporre il suo schema, Ferrer ha attinto alcune idee dal capitolo dedicato alla Pentecoste nella Legenda aurea di Iacopo da Varazze ( $†$ 1298), un testo da lui spesso utilizzato ${ }^{27}$. Lo si vede in tutta chiarezza confrontando le note sulla concordia fraternalis e una delle sette caratteristiche dei discepoli che, secondo la Legenda aurea, li rendeva idonei a ricevere lo Spirito. Entrambi i testi utilizzano l'immagine del corpo che deve restare unito per essere vivificato, richiamano Atti 2,1 e, soprattutto, citano il medesimo responsorio della liturgia del giorno ${ }^{28}$.

\section{LA PREDICA DI LA MORALEJA}

La prima testimonianza dell'uso effettivo di questo schema è costituita dalla reportatio latina della predica tenuta da Ferrer verosimilmente

${ }^{26}$ In riferimento alla strofa citata: "Inde dicitur annus iubileus, id est remissionis, et significat octavam diem resurrectionis in qua omnes liberati erunt de servitute dyaboli et hec est mistica, id est figurativa sunt, patrata, id est completa, per acto, id est completo tempore Pasce sacro numero dierum in quinquagenario, quo numero fit remissio in lege Moysi"; Expositio 1488 , f. 52v. L'identificazione tra la Vergine e la doctix di Sapienza 8,4 è tradizionale nei sermoni mariani, e serve principalmente a sottolineare il ruolo della Madonna nel rivelare gli eventi e i misteri legati alla nascita e infanzia di Gesù; si veda ad esempio Bartolomeo da Breganze 1993 , pp. 74, 105, 205, 412, 817.

${ }^{27}$ Perarnau 1999a, p. 58 e, con riferimento ad altri sermoni catalani, Catálan 2013,pp. 361-370.

28 "Secundo dilectione uniti, quod notatur in hoc quod dicitur erant omnes pariter. Erant enim eis cor unum et anima una. Sicut enim spiritus hominis non uiuificat membra corporis nisi unita, sic nec spiritus sanctus membra spiritualia. Et sicut ignis lignorum diuisione extinguitur, sic spiritus per discordiam in hominibus; et ideo cantatur de apostolis: Inuenit eos concordes caritate et illustrauit eos inundans diuinitas deitatis"; Iacopo da Varazze, Legenda aurea, ed. Maggioni 1998, p. 564. Nel testo si ritrovavano anche altri due dei rimandi agli inni liturgici utilizzati da Ferrer (ivi pp. 564 e 566). 
nella Pentecoste del 1411 a La Moraleja, in Castiglia ${ }^{29}$. Lo schema di Perugia è seguito con grande fedeltà ad eccezione dei riferimenti liturgici che qui non trovano spazio, per decisione del predicatore o forse del reportator che, in alcuni punti, lascia trasparire un approccio selettivo rispetto alla performance di Ferrer ${ }^{30}$. Il testo permette comunque di farsi un'idea della modalità con cui lo scarno schema del 1407 era sviluppato dal pulpito. Se nel manoscritto di Perugia si dice che tra gli apostoli c'era concordia fraternalis, davanti agli ascoltatori di La Moraleja Ferrer spiega come nei giorni tra Ascensione e Pentecoste la comunità primitiva avesse sanato le fratture avvenute tra la Passione e la Risurrezione: gli apostoli chiedono perdono alla Vergine per aver abbandonato -e rinnegato, nel caso di Pietro- suo figlio; Tommaso petit veniam per non aver creduto agli altri apostoli; essi si scusano con le pie donne per aver considerato deliramenta il loro annucio la mattina di Pasqua ${ }^{31}$. Di questo non c'era traccia nello schema di Perugia e neanche in Iacopo da Varazze, la fonte di questa sezione.

La reportatio lascia così intravedere il modo con cui Ferrer arricchisce e movimenta la descrizione della comunità apostolica. È questo un aspetto ancora più evidente nello sviluppo dell'audientia doctrinalis, dove l'ascolto della predicazione della Vergine è anticipato dal racconto dell'interrogarsi degli apostoli sul perché lo Spirito, promesso loro da Cristo, sembri tardare. A partire dalla domenica dopo l'Ascensione, ogni giorno gli apostoli ipotizzano una ragione per cui possa essere quello il momento giusto per la discesa dello Spirito. Lo schema non è però pienamente sviluppato, come invece sarà in versioni successive del sermone. Infatti, dopo aver indicato per la domenica il nesso con la Risurrezione e per lunedì quello con la creazione del firmamento (cfr. Genesi 1,6), il predicatore -o forse il reportator- si accontenta di dire: et sic de aliis diebus septimane, passando alla notte di Pentecoste, quando gli apostoli ormai disorientati si appellano alla Vergine che predica loro e li esorta a pregare. Dividendosi in tre gruppi (gli apostoli, i discepoli, le donne), la preghiera si svolge come una sorta di ufficio liturgico, in cui ciascun gruppo - guidato rispettivamente da Pietro, Lazzaro e la Maddalena- prega con uno specifico versetto biblico, secondo uno schema descritto con maggior

\footnotetext{
${ }^{29}$ Ferrer, Sermonario, ed. Gimeno, Mandingorra 2002, pp. 295-298 (ringrazio Tomàs Martínez Romero che mi ha procurato le fotocopie di questo testo). La reportatio è contenuta in Valencia, Real Colegio Seminario del Corpus Christi, f. 57v-58v. Su questo manoscritto Perarnau 1999c. Sul ciclo castigliano Cátedra 1994; Vidal 2010.

${ }^{30}$ Presentando l'abstinentia degli apostoli, Ferrer annuncia che "de hoc computabo vobis pulcram ystoriam", ma il reportator annota solo brevi citazioni tratte da Marco 2,18-20; Ferrer, Sermonario, ed. Gimeno, Mandingorra 2002, p. 295. Sul filtro operato dai reportatores, Rusconi 1989.

${ }^{31}$ Ferrer, Sermonario, ed. Gimeno, Mandingorra 2002, p. 296.
} 
ampiezza nelle successive reportationes. Sarà però la voce della Vergine, a cui tutti chiedono di unirsi loro nella preghiera a suscitare la pronta discesa dello Spirito.

A chiusura del sermone, Ferrer elenca quattro grazie concesse agli apostoli: scientia in intellecto, eloquentia in lingua, caritas in corde, stabilitas et firmitas in corpore. Esse trovano riscontro per formam in qua aparuit Spiritus, venendo visualizzate dal fuoco che illumina, ha forma di lingua, scalda, riempie la casa.

\section{LA REPORTATIO DI TORTOSA}

La seconda testimonianza dell'utilizzo di questo sermone è costituita dalla reportatio di una predica fatta da Ferrer a Tortosa, con tutta probabilità nel 1413, e contenuta nel manoscritto 477 della Biblioteca de Catalunya di Barcellona $^{32}$. Stando alla datazione proposta da Josep Perarnau, il sermone si collocherebbe nel contesto della disputa di Tortosa tra giudei e cristiani, promossa da Benedetto XIII, animata dal neo-convertito Jerónimo de Santa Fe e alla quale non fu estraneo il proselitismo operato da Ferrer attraverso una predicazione dai toni fortemente antigiudaici ${ }^{33}$. Il domenicano si sarebbe recato apposta a Tortosa per Pentecoste, così da fare sentire la sua voce in tale solenne festa ${ }^{34}$. Nel sermone, in realtà, traspare poco del contesto polemico che accompagnò la disputa, a meno di non volere leggere in tale senso l'esordio, dove Ferrer insiste nel chiamare i cristiani "veri figli di Israele" in quanto detentori della "vera credenza"35. Forse anche l'insistenza sulla discesa dello Spirito in forma di colomba al momento del battesimo nel Giordano implicitamente ribadiva come Gesù fosse il Messia - uno dei punti discussi nella disputa in corso $^{36}$.

Rispetto allo schema di Perugia e alla reportatio di La Moraleja, gli elementi di novità principale sono due. Il primo è il lungo prologo,

${ }^{32}$ Perarnau 1985, pp. 243-244 (datazione) e 315-326 (edizione).

${ }^{33}$ Santonja 2012. Su Ferrer e gli ebrei Vidal 2010; Losada 2015.

${ }^{34}$ Perarnau 1985, pp. 245-246, sottolinea che la presenza di Ferrer "responia a un designi, també polític, molt consciement formulat".

35 "Volets, vosaltres christians, qui sots dit vertaders fill de Israell, qui veets Déu per fe e vertadera creença (car açò vol dir Israel), que l'Esperit Sant vinga e habit ab vosaltres? Aparalats-li los vostres coratges, e servit a en tot soll, e ell desliurar-vos ha de mans dels philisteus" [1 Samuele 7,3] (...). O Israeli, ço és, o cresta! Ve·t que l'Esperit Sant, qui és Déu, qui és subirana consolació, e dolç aconsolador, vol venir a tu"; ibidem, p. 316.

${ }^{36}$ Ibidem, p. 318. La sezione sulla forma di colomba dello Spirito è attinta da Iacopo da Varazze, Legenda aurea, ed. Maggioni 1998, p. 552. 
teologicamente impegnato, dove si insiste sulle tre occasioni in cui Gesù dona lo Spirito agli apostoli, sempre a servizio della predicazione: prima della Passione, per predicare nella missione in Giudea (Matteo 10); dopo la Risurrezione, per dare loro autoritat de preïcar per lo món universal e per comprendere delle Scritture (Luca 24); a Pentecoste, quando le lingue di fuoco mostrano come havien a denunciar e preïcar ley fogajant e scalphada, ço és, ley de amor e de dilecció, visualizzando il dono di comprendere e parlare tutte le lingue ${ }^{37}$. La fonte utilizzata è ancora la Legenda aurea, dalla quale sono attinti sia la distinzione tra missione invisibile e visibile dello Spirito, sia l'enumerazione dei momenti in cui lo Spirito è stato dato agli apostoli ${ }^{38}$. La seconda differenza è che il corpo del sermone riduce da quattro a tre le disposicions necessarie per ricevere lo Spirito: abstinència corporall, oració afectuall, concòrdia fraternall. Il tema dell'ascolto della dottrina sarà recuperato nella parte conclusiva del sermone.

$\mathrm{Nel}$ descrivere le tre disposizioni ritornano, nella stessa sequenza, gli elementi annotati nello schema del 1407. La reportatio permette così di decodificare le connessioni che restavano solo implicite nel testo di Perugia. Ad esempio, se era prevedibile che il legno secco che prende fuoco rappresentasse chi, liberatosi da peccati e vizi attraverso digiuni e veglie, è pronto a ricevere lo Spirito, meno scontata è l'interpretazione delle ossa aride come le persone sante e devote che sorreggono il mondo con il loro buon esempio, così come le ossa sorreggono l'intero corpo $^{39}$. Mentre si rimanda all'esempio dei discepoli in attesa della Pentecoste, non c'è (o non è stato registrato) il riferimento all'inno Iam Christus. A differenza del 1411, l'effettivo uso dei testi liturgici è però confermato trattando le altre due disposizioni ${ }^{40}$.

Dopo aver trattato le tre disposicions, Ferrer annuncia che finirà con una breve istòria. È qui che, in parte, si recupera il tema dell'ascolto della predicazione. Nello schema perugino si indicava come la Vergine avesse istruito i discepoli sulle ragioni dell'apparente ritardo della discesa dello Spirito. Nella predica di Tortosa si racconta come la comunità riunita

\footnotetext{
${ }^{37}$ Perarnau 1985, pp. 318-320.

${ }^{38}$ Iacopo da Varazze, Legenda aurea, ed. Maggioni 1998, pp. 552 e 556-558. Il triplice invio dello Spirito sugli apostoli si trova già nella Glossa ordinaria a commento di Atti 2,1.

39 "E per ço, dix lo propheta: Ossa arida, audite verbum Dei. Ecce ego introducam in vos Spiritum et vivetis, Ezechiel, XXXIII capitulo [37,4], o ossos sechs, ço és, o persones sanctes e devotes, qui, axí com los ossos sostenen lo cos, axí vosaltres per eximpli de vostra bona vida sostenits lo món, e sots sechs per dejunis e per abstinències de delits e de ples corporals, oyts e scoltats la paraula e la promissió de Déu...”; Perarnau 1985, pp. 320-321. Il tema manca nel 1411.

40 "E de açò canta vuy la sglésia: Dum ora cuntis tertia... (...). E per ço, canta la sancta sglésia vuy: Advenit ignis divinus (...) Invenit eos concordes karitatis..."; ibidem, pp. 322 e 324 (segue parafrasi in volgare).
} 
nel cenacolo si preparasse con digiuni, preghiere e unita nell'amore (le tre disposizioni discusse nel sermone) e come nei giorni tra Ascensione e Pentecoste Maria predicasse ad apostoli e discepoli:

la subirana doctoressa e graciosa mestra, la sagrada verge Maria, los féu cascun dels deu jorns una meravellosa collació, excitant-los e confirmant-los en la gràcia e amor del seu reverent fill, e en la promissió a ells feta del eveniment del Sant Sperit. E preïcant-los ella axí cascun dia, vinch lo un dia, ço és, lo dia de Cinquagesma... ${ }^{41}$

Se sul contenuto della predicazione quotidiana della Vergine non si dice altro, l'attesa nel giorno di Pentecoste, seguendo l'invenzione narrativa già riscontrata nel 1411, è descritta come una liturgia comunitaria che mette in scena, in maniera ordinata, $\mathrm{i}$ tre gruppi che componevano la Chiesa primitiva. All'ora prima, gli apostoli, desiderosi di ricevere lo Spirito, si riuniscono nel cenacolo e aquí, ficants los genols en terra e alçant les mans vers lo cell, dix ab làgrimes mosènyer sant Pere en nom de tots: 'Cor mundum crea in me, Deus, et Spiritum Sanctum innova in visceribus meis' (Salmo 50,12). Alla preghiera si aggrega Lazzaro -posto qui, in maniera sorpredente, a capo del gruppo dei 72 discepoli (cfr. Luca 10,1-10) e degli amichs de Jhesuchrist- usando un altro versetto biblico: Sensum tuum quis sciet, Domine, nisi tu dederis sapientiam et miseris Spiritum Sanctum tuum de altissimis? (Sapienza 9,17). Il terzo gruppo è quello delle donne, guidate dalla Maddalena, inflamada amadora de Jhesuchrist, la quale prega con un altro passo del Miserere: Redde mihi letitiam salutaris tui et spiritu principali confirma me (Salmo 50,14). Con perfetto tempismo, la Madonna all'ora terza si pone in mezzo ai 120 discepoli (un numero su cui Ferrer insiste) e agenolada, ab les mans e ab lo uls elevats vers lo cell, dix: Emitte Spiritum tuum et creabuntur et renovabis faciem terre (Salmo 103,30). A queste parole risponde -come nel racconto biblico-il tuono dall'alto e la discesa delle lingue di fuoco che si posano sopra i discepoli. Ricolmi dello Spirito, tutti esclamano con gioia $O$ quam bonus et quam suavis est, Domine, Spiritus tuus in nobis, prima che gli apostoli escano dal cenacolo e comincino a prehicar $e$ a denunciar Jhesuchrist ${ }^{42}$. Dopo la messa in scena del racconto di Pentecoste, la predica si chiude con una rapida rassegna degli effetti dello Spirito sulla comunità primitiva, utilizzando uno schema diverso da quello del $1411^{43}$.

\footnotetext{
${ }^{41}$ Ibidem, p. 325. Non si menziona l'attesa lungo la settimana, accennata nel 1411.

42 Ibidem, p. 326. Il testo rielabora Sapienza 12,1 come in uno dei responsori della liturgia: http://cantusindex.org/ [consultato: 18/03/2018].

${ }^{43}$ Gli effetti sono: remissione dei peccati; abbondanza dei sette doni dello Spirito; conoscenza delle Scritture; profezia; dono delle lingue; fervore per predicare.
} 


\section{LA REPORTATIO DEL 1416}

Il manoscritto 279 della Biblioteca della cattedrale di Valencia (281 nella antica numerazione), pubblicato da Josep Sanchis Sivera, contiene una reportatio che attesta l'uso del sermone Repleti sunt omnes per Pentecoste, con ogni probabilità ad Albi nel $1416^{44}$.

Rispetto alla reportatio di Tortosa, il prologo è ridotto a una breve disquisizione sui tre momenti in cui gli apostoli ricevono lo Spirito, mentre il corpo del sermone si concentra sui preparativi (apparellaments) fatti dai discepoli dopo l'Ascensione, ritornando allo schema a quattro membri. Lo sviluppo dei primi tre punti ricalca da vicino quello del 1413, riprendendo ad esempio l'interpretazione delle ossa come simbolo delle persone che con le loro preghiere sostengono, dall'interno, tanto il corpo tutto della christianitat quanto la vita di una vila, una ciutat, hun realme ${ }^{45}$.

Le espansioni toccano l'applicazione dei contenuti morali agli ascoltatori e la messa in scena degli episodi biblici citati. Parlando della preghiera, ad esempio, Ferrer inserisce una sarcastica descrizione delle orazioni dette distrattamente, mentre si è intenti in altre occupazioni, dal vestirsi al dare ordini alla serva, al sistemarsi le ciglia davanti allo specchio:

Lo segon apparellament (...) és iminència spiritual (vol dir oració, mas yo dich iminència spiritual), no solament parlant de boqua Pater noster, axí com vosaltres, quan feu oració, e com quan vos vestiu e preneu la camisa per lo matí, 'Pater noster' a la una mànega, e 'xa xa xa, Marieta, posa l'olla, Pater noster', (o, que bella oración!), e quan vos botonau 'Aue Maria, ¡eee!, gracia ple$n a$, xa xa xa', no val res: altres Patersnostres de lançol, ni val ni nou; e vosaltres, dones, ¿com feu oració? 'Senyor, quan me lligue e'm estire les celles, e prench lo mirall', 'Aue Maria, xa xa xa', no val res aytanpoch; mas la oració que·s fa axí devotament agenollat, e levant la pensa en Déu ${ }^{46}$.

Il brano è spesso citato per esemplificare l'efficacia di Ferrer nel descrivere scene tratte dalla vita quotidiana, costruite usando un tono "popolare" e soffermandosi su gustosi dettagli ${ }^{47}$. L'insistenza sulla necessità di una preghiera fatta con devozione e non in mezzo ad altre occupazioni è, del resto,

\footnotetext{
${ }^{44}$ Ferrer, Sermons, ed. Sanchis 1932-1934, vol. I, pp. 133-146. Sulla datazione del sermone Riquer 1963-1964.

${ }^{45}$ Ferrer, Sermons, ed. Sanchis 1932-1934, vol. I, p. 135.

${ }^{46}$ Ibidem, p. 137.

${ }^{47}$ Fuster 1954, p. 41; Delcorno 2006, p. 278.
} 
un elemento ricorrenti nei sermoni di Ferrer e nella sua idea di acculturazione religiosa dei laici ${ }^{48}$.

Nella stessa direzione va un altro ampliamento del sermone. La reportatio di Tortosa, trattando della preghiera degli apostoli, inseriva una breve quaestio sul perché gli apostoli chiedano quanto era già stato loro promesso $^{49}$. Nel 1416 la risposta si fa più articolata, affrontando il tema della predestinazione e applicandolo alle norme di vita delle diverse fasce sociali. Ferrer prima chiarisce come, contrariamente a quanto credono i suoi ascoltatori, nel libro dei predestinati non siano scritti solo i nomi di salvati e dannati, ma due elenchi -scritti in lettere d'oro e in tinta negra-che comprendono le motivazioni che portano uno a essere salvato o dannato. Se la salvezza di un re può dipendere dal compiere la giustizia, ai potenti è chiesto di trattare axí al pobre com al rich, ai religiosi di osservare la propria regola, ai ricchi di compiere le opere di misericordia, là dove per alcuni si registra solo l'avere fatto penitenza. Per i dannati avviene il contrario, come è annotato per i futuri predicatori dicendo "dic ut supra, per lo contrari".

Trattando invece della concordància fraternal, Ferrer inserisce -come già a Tortosa ${ }^{50}$ - l'elenco di casi in cui gli apostoli, durante il ministero terreno di Gesù e prima dell'incontro con il Risorto, si mostrarono divisi tra loro. Il predicatore drammatizza qui il racconto evangelico, immaginando la discussione per stabilire chi fosse il più importante degli apostoli ( $c f r$. Luca 22), espandendo così l'elenco di casi discussi a La Moraleja. A Pietro che ritiene non ci sia discussione, visto che Gesù ha detto che yo seré papa e major de vosaltres, e prelat de tot lo món, replica Andrea ricordando come sia stato lui il primo dei discepoli (Giovanni 1,35-40), ma a entrambi si oppone Giovanni che rivendica la superiorità della propria condizione verginale rispetto a discepoli che, in quanto sposati e con figli, sono gent carnal $^{51}$. La messa in scena delle dispute tra discepoli sottolineava la necessità di essere pronti a ricucire gli strappi all'interno della comunità civile ed ecclesiale -un tema tanto più sentito negli ultimi, turbolenti anni dello scisma.

Una simile tecnica di drammatizzazione è utilizzata trattando la audiencia doctrinal. Dopo il richiamo all'immagine della pietra focaia -ricordato all'inizio dell'articolo- e alla Vergine quale 'predicatrice' degli apostoli, Ferrer annuncia di voler concludere con una práctica, cioè-secondo la definizione datane da Pedro Cátedra- una representación o puesta en práctica de los consejos o discusiones teológicas planteadas, revitalizada

\footnotetext{
${ }^{48}$ Delcorno 2006, pp. 276-279.

${ }^{49}$ Perarnau 1985, pp. 322-323.

${ }^{50}$ Ibidem, pp. 323-324.

${ }^{51}$ Ferrer, Sermons, ed. Sanchis 1932-1934, vol. I, p. 141.
} 
siempre por medio de la escenografia, la acción y la caracterización de los personajes, una dramatización mental utilizzata in particolare per avvicinare gli episodi della Scrittura agli ascoltatori ${ }^{52}$.

La reportatio arricchisce il racconto già inscenato a Tortosa, aggiungendo ulteriori elementi, il più significativo dei quali è l'invenzione dell'attesa dubbiosa degli apostoli che, nei giorni dopo l'Ascensione, come già accennava la reportatio del 1411, si interrogano su quale possa essere il momento propizio per la discesa dello Spirito. Prendendo la parola a turno, ciascuno di loro scorge le possibili connessioni tra i giorni della settimana e il dono dello Spirito. Oltre a descrivere il crescente sconcerto degli apostoli per l'apparente ritardo dell'adempiersi delle promesse, il racconto ha una funzione didattica, enumerando le qualità e i compiti del Paraclito ${ }^{53}$. Si tratta versosimilmente di un cambiamento dello schema originale, perché la Vergine non predica più loro ogni giorno, come affermavano lo schema di Perugia e la reportatio del 1413 e, in realtà, come accennava subito prima lo stesso testo del 1416. In questa increspatura logica si può cogliere il segnale di un predicatore che trasforma i propri schemi, lasciando a volte visibili i segni del suo intervento.

La soluzione ai dilemmi degli apostoli è offerta dalla Vergine che, anche in questo testo, impartisce una breve catechesi sul giorno di Pentecoste. In questo caso però, usando il registro patetico e costruendo un racconto emotivamente più intenso, sono i discepoli che, ormai scoraggiati, si recano di notte dalla Vergine, supplicandola che sveli loro le ragioni del prolungarsi dell'attesa. Si ritorna poi a descrivere la preghiera nei tre gruppi guidati da Pietro, Lazzaro e la Maddalena, ai quali si aggiunge infine la Vergine, tanto che il tuono è descritto come una risposta dal cielo alla sua preghiera. La discesa dello Spirito è qui descritta attraverso una serie di particolari cromatici: la luce del fuoco che invade il cenacolo e traspare dalle sue finestre, il cielo rosso sangue sopra la città, il fumo bianco come incenso che esce dalla casa. Ferrer sembra voler rendere visibile la scena, associando poi ai colori significati simbolici ${ }^{54}$. Dopo una menzione della predicazione dell'apostolo Pietro ai giudei, si richiama l'inno Beata nobis gaudia, come nel manoscritto di Perugia ${ }^{55}$.

${ }^{52}$ Cátedra 1994, pp. 219-220.

${ }^{53}$ Per l'analisi di questa scena, vedi infra p. 94.

54 "E significave açò que·us diré: que·l recebiment del sant Spirit fo per mèrit de la passió de Jesuchrist, e per ço lo sol se mostrà royg; e les lengües del foch significaven com los apòstols havien a disputar, a preycar, a rahonar davant totes gens, philòsofs soptils e aguts, e reys, e prínceps; lo fum blanch significave la dolçor que ells sentien"; ibidem, p. 146. 139.

${ }^{55} \mathrm{La}$ reportatio registra anche le due ricorrenze dell'inno Iam Christus; ibidem, pp. $137 \mathrm{e}$ 


\section{Il TESTO STANDARDIZZATO: DRAMMATIZZARE LA PENTECOSTE}

Se già il manoscritto di Valencia raccoglieva le reportationes di Ferrer ordinandole secondo il calendario liturgico per facilitare i futuri predicatori, un passo ulteriore in questa direzione è rappresentato da una serie di manoscritti latini organizzati secondo i grandi cicli de tempore e de sanctis ${ }^{56}$. Del resto, nel 1454, durante il processo di canonizzazione, l'arcivescovo di Tolosa attestò come i sermoni di Ferrer non solo fossero stati raccolti tam in latino quam in vulgari da qualificati reportatores (teologi e giuristi), ma come la pratica di copiare, diffondere e utilizzare i testi di Ferrer fosse viva a più di trent'anni dalla sua morte ${ }^{57}$. L'approdo di questo processo è costituito dalle edizioni a stampa che, a partire dal Quadragesimale del 1482, cristallizarono i sermoni di Ferrer, rendendoli accessibili e consegnandoli alle generazioni seguenti.

L'ultima versione del sermone Repleti sunt omnes che prendiamo in esame proviene da questo tipo di fonti. Considererò qui l'editio princeps dei sermoni di Ferrer, pubblicata a Colonia da Heinrich Quentell nel 1485, confrontandola con uno dei manoscritti che contiene i suoi Sermones de tempore: Toulouse, Bibliothèque municipale, MS 345 (seconda metà del Quattrocento ${ }^{58}$. I due testi sono assai vicini, tanto da suggerire di considerarli insieme, anche se il manoscritto è a tratti più stringato e conserva alcune espressioni in volgare: ad esempio, a proposito della pietra focaia, parla di "similitudinem del foguey", definendo Cristo "lapis fogueyra"59.

Il sermone si apre con un nuovo prologo, dove si spiega come apostoli e discepoli non avessero la plenitudinem Spiritus prima di Pasqua e come questa gli fosse poi data in tre forme. La prima è la plenitudo scientie intellectualis, rappresentata dalla comprensione delle Scritture, concessa dal

\footnotetext{
${ }^{56}$ Su questi manoscritti: Perarnau 1999a, pp. 9-11; 1999d, p. 280.

57 "Plures valentes viri, theologi et juristae (...) promptam manum habentes ad scribendum sermonem, quem ipse magister Vincentius pronuntiabat, de verbo ad verbum tam in latino quam in vulgari integraliter scripserunt praefatos sermones plenos magna scientia, magnis auctoritatibus divinae scripturae, claros catholica doctrina fructuosa et salubri, sapida et redolenti mirabili movere corda et conscientias audientium, cujuslibet aetatis intelligibilis et cujuscumque status ecclesiastici vel mundani. Et dictae reportationes sive collectae sermonum (...) fuerunt ex post per multos scientificos transumptati sive scripti et ad diversas mundi partes transportati et multi praedicatores ex post usi sunt in praedicationibus suis dictis sermonibus catholicis et excellentibus et utuntur adhuc hodiernis temporibus"; Fages 1904, pp. 279-280.

${ }^{58}$ Perarnau 1999d, p. 280 indica il manoscritto come "el text paradigmàtic d'aquesta versió". $T$, con scarne indicazioni bibliografiche, è accessibile online: http://numerique.bibliotheque. toulouse.fr/ark:/74899/B315556101_MS_000345 [consultato: 06/03/2018]. Nel capolettera della prima carta, una miniatura, attribuita a Antoine de Lonhy $(\dagger c .1490)$, ritrae Ferrer mentre predica dal pulpito.

${ }^{59} T$, f. 150vb. Nel testo a stampa si trovano invece: "similitudinem de ignali" e "lapis ignitus". Sull'espansione dei riferimenti teologici nei sermoni a stampa, Perarnau 2011-2013, p. 532 .
} 
Risorto (cfr. Luca 24). La seconda è la potentia spiritualis che li legittima a operare in nome di Cristo, sciogliendo e legando i peccati (cfr. Giovanni 20). Infine, a Pentecoste, gli apostoli ricevono la plenitudo de eloquencia universali. Fornito questo quadro, il sermone introduce le quattro dispositiones necessarie a ricevere lo Spirito.

Nello svolgimento si ritrovano i punti già incontrati, fatta eccezione per i riferimenti agli inni liturgici. Vi sono, come prevedibile, variazioni. Rispetto alle reportationes sparisce l'interpretazione delle ossa come sostegno di tutto il corpo, mentre la descrizione della preghiera meccanica in mezzo ad altre occupazioni è appena accennata, riassumendola nella contrapposizione tra orationes e recitationes ${ }^{60}$. Si ritrova anche in questo testo l'excursus sulla predestinazione, in forme vicine alla reportatio di Albi, traendo però gli esempi dal catalogo dei vizi piuttosto che dalle diverse classi sociali ${ }^{61}$.

Se in diversi punti il tono del sermone è più levigato rispetto alle reportationes -ad esempio la discussione tra gli apostoli è ridotta a un breve accenno- resta invece ampia e vivace la messa in scena dell'attesa impaziente dello Spirito da parte dei discepoli. Per dare un'idea più precisa di come Ferrer unisca una drammatizzazione del racconto biblico, aperta a inventive soluzioni narrative, e una sofisticata catechesi sulle caratteristiche dello Spirito, riporto in maniera integrale la sezione sulla quarta dispositio, seguendo il testo del manoscritto di Tolosa e segnalando in nota alcune delle differenze più rilevanti riscontrabili nell'editio princeps ${ }^{62}$.

[f. 150vb] Quarta dispositio multum necessaria ad recipiendum spiritum sanctum est audiencia doctrinalis: audire libenter verba Dei. Non dico Ovidii vel aliorum poetarum ${ }^{63}$ etc. quia illi non habuerunt spiritum sanctum et quomodo darent vobis? Sed doctrina Dei illa dat spiritum sanctum, declaratur per similitudinem del foguey quia percussio lapidis ad ferrum excutit ignem in escam. Recte ita est de predicatione: lapis fogueyra est Christus Petra autem erat Christus, prima Corintheos $11[11,4]$-ferrum sive clavis est lingua predicatoris: Non vos estis qui loquimini, sed spiritus patris vostri, qui loquitur etc. Matthei 10 [10,20]; esqua est populus, nigra ex peccatis ${ }^{64}$. Accenditur autem igne spiritus sancti quando in prima vel secunda vel in tercia predicatione superbus vanus pomposus humiliatus proponit de cetero vivere simpliciter.

\footnotetext{
60 "aliqui orant que proprie non dicuntur orationes se quedam recitaciones, quando induuntur et mulieres quando ornantur"; $E$, f. NN2vb.

${ }^{61} E$, f. NN3rb.

${ }^{62}$ Nella trascrizione sciolgo le abbreviazioni e adatto la punteggiatura e le maiuscole secondo l'uso moderno.

${ }^{63}$ E: "Ovidii, Terencii, Virgilii et sic de aliis".

${ }^{64} E$ : "materia nigra est populus qui est niger ex peccatis".
} 
A modo ista esqua ${ }^{65}$ est ignita igne spiritus sancti, et sic de aliis peccatis. Autoritas: Adhuc eo loquente Petro verba hec, cecidit spiritus sanctus super omnes qui audiebant verbum eius; Actuum $10[10,44]$. Istam audienciam doctrinalem verbi Dei habuerunt apostoli, quia a die ascensionis citra quolibet die habebant sermonem. Et quis predicabat? ${ }^{66}$ Dico quod virgo Maria, quam Christus dimisit loco suo doctricem et magistram: doctrix, id est doctoresse, est discipline Dei et lectrix ${ }^{67}$ operum illius; Sapiencie 8 [8,4]. Christus autem ante suam ascensionem promisit mittere spiritum sanctum apostolis dicens: 'Expedit vobis ut ego vadam. Si autem non abiero paraclitus non veniet ad vos, [f. 151ra] si autem abiero mittam eum ad vos' [Giovanni 16,7], sed non dixit eis quando, scilicet quo die nec qua hora. Alia promissio fuit in die ascensionis, quando fuit in Monte Oliveti et debuit ascendere dixit: Iohannes quidem baptisavit aqua, vos autem basptisabimini spiritu sancto non post multos hos dies; Actuum primo [1,5]. Apostoli vero nescientes certum diem huius promissionis in die ascensionis et feria sexta in crastinum et sabbato expectaverunt credentes quod in illis diebus mitteret spiritum sanctum, sed transactis his tribus diebus, videntes quod Christus non miserat eis spiritum sanctum, ceperunt tristari et dicere adinvicem: 'O, et quando mittet?'. Respondit unus: 'Non dubitetis, quia cras que est dies dominica mittet; motivum quia tali die Deus formavit lumen ${ }^{68}$, divisit lucem a tenebris [Genesi 1,4]. Ideo rationabiliter tali die mittet nobis lucem spiritus sancti'. Et transivit dominica et non misit, et qualiter mirabuntur. Dixit alter: 'Die lune mittet, quia tali die fecit firmamentum et divisit aquas que erant super firmamentum ab aquis que < erant $>$ subter firmamentum [Genesi 1,7], tali ergo die mittet, quia modo indigemus firmamento fortitudinis ut dividamus aquas virtutum ab aquis viciorum'. Et non venit. Dixit alius: 'Die martis mittet, ratio quia tali die de terra produxit plantas ad fructificandum [cfr. Genesi 1,11-12], tali ergo die mittet ut possimus plantare fidem catholicam ut faciat $<$ fructus>'. Et expectantes, non venit. Dixit alius: 'In die mercurii mittet, quia tali die formavit solem, lunam et stellas sive planetas [cfr. Genesi 1,14-16], tali ergo die <mittet> ut creet in nobis solem caritatis, lunam spei, planetas virtutum'. Et non venit. Cogitate qualiter mirabantur et contristabantur. Dixit alius: 'Die iovis mittet nobis, ratio quia tali die ascendit ab aspectibus nostris. Ideo tali die consolabitur nos, quia illa die ex recessu eius fuimus desolati'. Et non venit. Dixit alius: 'Die veneris [f. 151rb] mittet, ratio quia tali die fuit passus et nos habuimus dolores sue passionis propter quod meruimus ut tali die daret nobis spiritum consolatorem'. Et non venit. Dixit alius: 'In sabbato vere mittet, ratio quia tali die fuimus dispersi que no sen tenia lun ab lautre, ideo tali die mittet

\footnotetext{
${ }^{65} E$ : "persona".

${ }^{66} E$ : "Si dicatur: Quis predicabat?".

${ }^{67} E$ : "electrix". $E$ risulta più corretto, ma lectrix può essere una variante intenzionale.

${ }^{68} \mathrm{~T}$ : "hominem". Correggo sulla base di $E$.
} 
ut sibi uniamur'. Et non venit ${ }^{69}$. Videntes autem quod sabbatum transivit et non venerat et iam perdiderant comptum dierum et timebant Iudeos: Erant enim congregati propter metum Iudeorum [Giovanni 20,19]. Cogitate rationabiliter quomodo erant tristes et desolati $^{70}$. Ideo omnes hodie simul congregati iverunt ad portam camere virginis Marie, expectantes quando exiret ut consueverat. Ipsa autem virgo surgebat in media nocte, iuxta illud media nocte surgebam ad confitendum tibi [Salmo 118,62], et dicebat officium suum. Quando autem virgo Maria exivit cameram et vidit omnes apostolos et discipulos flentes, dixit eis: 'Filii, quid est hoc? De quo fletis, quia filius meus modo regnat in celis etc.?' Et dixerunt sibi: 'Benedicta domina ${ }^{71}$, filius vester promisit nobis mittere spiritum, dicens quod non dimitteret nos orphanos [cfr. Giovanni 14,18] inter Iudeos, modo autem sunt decem dies quod ascendit et nondum misit nobis spiritum sanctum'. Respondit virgo Maria: 'Non dubitetis ${ }^{72}$, quia filius meus non habet conditiones hominum mundanorum, qui in paupertate congnoscunt amicos etc. ${ }^{73}$. Sed dicam vobis rationem quare non misit. Nescetis vos quod quando Deus extraxit populum Israel de terra Egipti de captivitate 50 die descendit in forma ignis in monte Synai dando legem?' Et apostoli virgo benedicta: 'Bene scimus illud!'. Et virgo Maria: 'Illud fuit figura. Filius meus in die resurrectionis extraxit de captivitate Egipti ${ }^{74}$ omnes sibi credentes et obedientes. Hodie autem est quinquagesimus dies a resurrectione, filii mei. Ideo hodie mittet spiritum sanctum. [f. 151va] Hodie ponatis vos omnes in oratione, quia que a Deo sunt ordinata sunt <orationibus impetranda> ${ }^{\prime}$. Tunc Petrus apostolus cum aliis apostolis posuerunt se ad unam partem et quilibet orando dicebat: 'Cor mundum crea in me Deus, et spiritum rectum innova in visceribus meis'. David regestravit in psalmo 50 [50,12]. Lazarus autem cum aliis 72 discipulis ad aliam partem et flexis genibus orando dicebant: 'Sensum tuum quis sciet nisi tu dederis sapientiam et miseris spiritum sanctum de altissimis?' Sapientia 7 [9,17]. Maria Magdalena et alie sancte mulieres flexis genibus orando dicebant: 'Redde michi leticiam salutaris tui et spiritu principali confirma $m e$ ' [Salmo 50,14]. Inde apostoli dixerunt virgini Marie: 'Virgo benedicta, etiam supplicamus orete, quia statim audiet vos filius vester'. Et erat tunc hora tercia. Tunc virgo Maria flexis genibus oravit dicens: 'Emitte spiritum tuum et creabuntur et renovabis faciem terre'; 103 [Salmo 103,30]. Nota: creabuntur, scilicet virtutes

${ }^{69} E$ : "dispersi ita quod unus non remansit cum alio. Ideo hodie ut sibi uniamur mittet".

${ }^{70} \mathrm{Sul}$ margine di $T$, un lettore ha inserito una manicula.

${ }^{71} \mathrm{E}$ : "Benedicta mater nostra".

${ }^{72} E$ : "Respondit virgo: Non dubitetis, filii mei...". Qui e in altri punti $T$ ha virgo Maria, E solo virgo.

${ }^{73} \mathrm{E}$ : "congnoscunt amicos et quando sunt in propseritate et honore non curant nec recordantur de eis". Sulla base di $E$, correggo $T$ dove si legge: "in paupertate non cognoscunt".

${ }^{74} \mathrm{E}$ : "inferni".

${ }^{75}$ Integro $T$ sulla base di $E$, dove si legge: "quia que a Deo ordinata sunt, precibus et devotis orationibus sunt impetranda". 
infuse dona gratie et perfectiones, et renovabis faciem terre, scilicet per predicationem apostolorum et discipulorum tuorum. Facta oratione ab sancta Maria virgine, subito factus est de celo sonus [Atti 2,2] magnus adinstar tonitrui non terrentis sed delectantis, fuit quasi vox responsiva virgini Marie; quem sonum audierunt Iudei qui erant in Iherusalem et subito ad instar venti descendit de celo et ille sonus replevit totam domum ubi erant apostoli [Atti 2,2] et tota domus repleta est igne. Iudei autem ad dictum sonum concurrentes viderunt ignem clarissimum per fenestras, sed ille ignis non comburebat sed illuminabat et videbant fumum album ad instar fumi incensi, et ille ignis divisit se ad instar linguarum seditque supra singulos eorum et repleti sunt omnes spiritu sancto [Atti 2,3-4]. Multi sancti de una gutta mellis [f. 151vb] spirituas sancti inebriati sunt dulcedine guadii, quanto magis hodie apostoli qui tota la bresqua del sprit sant receboren et dicebant: ' $O$ quam suavis est Domine spiritus tuus in nobis'; Sapientia $12^{76}$. Tunc fuit completa prophetia Ioelis dicens: In diebus illis effundam spiritum meum super servos meos et ancillas meas, et dabo prodigia in celo et in terra, sanguinem et ignem et vaporem fumi. Ioelis 2 [2,2930]. Nota: dabo prodigia in celo, quia tunc celum videbatur super Ierusalem sanguinolentum innuens quod merito passionis Christi mittebatur spiritus sanctus apolostolis. Et in terra apparuit ignis clarus qui ab omnibus poterat videri in domo ubi erant apostoli. Et vapores fumi ad instar incensi etc. Explicit sermo, Deo

In questo testo, è pienamente sviluppata, come nella reportatio del 1416, l'idea narrativo-catechetica degli apostoli che, passati tre giorni dall'Ascensione, iniziano a rattristarsi e, prendendo la parola a turno, cercano di incoraggiarsi l'un l'altro individuando le ragioni teologiche per cui ciascun giorno può essere quello in cui giungerà lo Spirito. L'attesa si prolunga per l'intera settimana, ripetendo lo stesso schema narrativo ogni giorno. È evidente la funzione narrativa, efficace nel descrivere il crescente smarrimento degli apostoli. Ferrer esorta ripetutamente gli ascoltatori a immaginare lo sconcerto dei discepoli: trascorso il mercoledì si dice Cogitate qualiter mirabantur et contristabantur e passato il sabato si insiste dicendo Cogitate rationabiliter quomodo erant tristes et desolati. Il sermone non va solo capito, ma seguito in maniera emotivamente partecipe. Il racconto, inoltre, sviluppa un sofisticato schema catechetico, in quanto ciascuna ipotesi fatta dagli apostoli mette

${ }^{76}$ L'intera frase ("Multi [...] 12") è assente in $E$, che la cita in un sermone per la Natività della Vergine (vedi nota 81): "Et ceperunt loqui quasi dicerent: $O$ quam bonus et suavis est Domine spiritus tuus nobis; Sapientie duodecimo. Cogitate hic quia multi sancti de una gutta mellis spiritus sanctus sunt inebriati; quantomagis apostoli qui totam virtutem spiritussancti receperunt"; Ferrer 1485, vol. III, f. S7v. Il concetto è presente nella reportatio del 1416: "quan una persoina devota sent una gota de consolació, està quasi tota arrapada; e, donchs, ¡quant més ells, que havien rebuda tota la brescha!"; Ferrer, Sermons, ed. Sanchis 1932-1934, vol. I, p. 146. 
in luce una delle caratteristiche dell'azione dello Spirito, collegandolo con gli eventi della Creazione e della Passione. La sequente tabella sintetizza il percorso didattico proposto da questo schema narrativo.

\begin{tabular}{|l|l|l|}
\hline GIORNO & RIFERIMENTO & EFFETTO \\
\hline Domenica & Creazione luce & Spirito illumina \\
\hline Lunedì & $\begin{array}{l}\text { Creazione firmamento divide le } \\
\text { acque }\end{array}$ & $\begin{array}{l}\text { Spirito fortifica e rende capaci di dividere vizi } \\
\text { e virtù }\end{array}$ \\
\hline Martedì & Creazione piante che fruttificano & $\begin{array}{l}\text { Spirito dato per piantare e far fruttificare la } \\
\text { fede }\end{array}$ \\
\hline Mercoledì & Creazione sole, luna e stelle & Spirito infonde carità, speranza, virtù \\
\hline Giovedì & Ascensione di Cristo & Spirito consola \\
\hline Venerdì & Passione Cristo e dolore apostoli & Spirito consola \\
\hline Sabato & $\begin{array}{l}\text { Apostoli dispersi dopo morte di } \\
\text { Gesù }\end{array}$ & Spirito unisce \\
\hline
\end{tabular}

Il prologo del sermone affermava che gli apostoli, dopo Pasqua, avevano la plenitudo scientiae che permetteva loro di interpretare le Scritture. In questa prospettiva, le loro affermazioni descrivono correttamente l'azione dello Spirito, valorizzando in particolare la sua funzione creatrice, un tema adatto al tempo liturgico in cui si cantava il Veni creator Spiritus ${ }^{77}$. Sarà però solo l'intervento della Vergine a offrire la giusta chiave ermeneutica, spostando l'interpretazione dai giorni della settimana al computo del tempo tra la Risurrezione e la discesa dello Spirito che rinnova quanto annunciato, in figura, dai 50 giorni trascorsi tra la liberazione dalla schiavitù d'Egitto e il dono della legge sul Sinai. Quello della Vergine, la doctrix lasciata da Cristo in sua assenza, è un breve sermone dove si cura non solo il contenuto didattico, ma anche la strategia comunicativa, impiegando alcuni degli strumenti tipici dei predicatori, come le domande rivolte agli ascoltatori. Segue la preghiera, capeggiata da Pietro, Lazzaro e la Maddalena, secondo lo schema riscontrato fin dalla reportatio del 1411. La loro invocazione resterà però senza effetti fino a quando non si aggiungerà l'intercessione potente della Vergine che flexis genibus oravit dicens: 'Emitte spiritum tuum et creabuntur et renovabis faciem terre', un rinnovamento -si annota ancora una volta- che sarà operato dalla predicazione. Col riferimento al tuono dall'alto, il racconto confluisce in quello di Atti, sottolineando come la discesa dello Spirito sui discepoli compia la profezia di Gioele.

\footnotetext{
${ }^{77}$ Sul rimando al Veni creator nei sermoni dell'epoca, Delcorno 2019.
} 


\section{CONCLUSIONE}

I testi analizzati permettono di vedere come Ferrer, nel presentare agli ascoltatori le modalità per preparsi a ricevere lo Spirito, elabori uno schema base e lo ripeta con costanza lungo gli anni. Ferrer non è però un usignolo meccani$\mathrm{Co}^{78}$, ma procede variando gli elementi e, in particolare, elaborando l'esordio e la conclusione del sermone, ovvero due punti strategici per ciascuna predica. Inoltre, le fonti analizzate testimoniano la dimensione europea di un sermone che, nelle sue diverse forme, il predicatore valenziano utilizzò in Italia, in Castiglia, in Aragona, in Linguadoca e, probabilmente, negli altri paesi toccati dal suo itinerario. L'instancabile azione di Ferrer lasciò dietro di sé una scia di manoscritti (Perugia, Valencia, Barcellona, Tolosa) che -riflettendo il multilinguismo della predicazione del tempo- raccolgono, rielaborano e sistematizzano il sermone di Pentecoste tanto in volgare quanto in latino. Questa circolazione europea sarà potenziata su larga scala dal successo dei sermoni a stampa di Ferrer che, dal 1485 a fine secolo, conobbero ben dodici edizioni, pubblicate in centri come Colonia (1485; 1487), Basilea (1488), Strasburgo (1489; 1493), Lione (1490; $1493 ; 1497 ; 1497 ; 1499)$, Norimberga (1492) e Venezia $(1496)^{79}$.

Venendo alle strategie comunicative del sermone, si riscontra l'abilità di Ferrer nel miscelare catechesi e invenzione narrativa, in particolare nella descrizione dell'attesa degli apostoli dove media per i suoi ascoltatori il racconto biblico, arricchendolo e, in un certo senso, piegandolo alle proprie esigenze. La descrizione dei discepoli che, perplessi, si interrogano sulle ragioni del prolungarsi dell'attesa e poi, nel cuore della notte, in lacrime, si rivolgono alla Vergine non sembra infatti avere -per quanto si è potuto vedere- specifici antecedenti e, almeno in questa forma, risulta una sofisticata invenzione comunicativa di Ferrer ${ }^{80}$. La drammatizzazione del racconto biblico non era però fine a se stessa e si accompagnava ad altre forme di accesso alla Scrittura, come la spiegazione di alcune profezie anticotestamentarie -le ossa di Ezechiele o l'annuncio di Gioele- che contribuivano a sedimentare negli ascoltatori una familiarità, seppure mediata e frammentaria, con la Bibbia. Dal punto di vista dei contenuti, il discorso mischiava toni 'popolari', come quelli usati nell'esortazione alla preghiera, a spunti teologici più impegnativi, come l'accenno al tema della predestinazione.

${ }^{78}$ Riprendo l'espressione con cui Roberto Longhi definiva l'opera, a suo avviso ripetitiva, del pittore Bernardo Daddi; Longhi 1974, p. 95.

${ }^{79}$ Seguo l'Incunabula Short Title Catalogue curato dalla British Library (http://data.cerl.org/ istc/_search [consultato: 08/03/2018]).

${ }^{80}$ Ho preso in considerazione: la Legenda aurea, lo Speculum humanae salvationis, la Vita Christi di Ludolfo di Sassonia, le Meditationes vitae Christi. 
Inoltre, indicando come modello per tutti i fedeli la comunità delle origini e descrivendone (o inventandone) la preghiera comunitaria, Ferrer sottolinea, almeno implicitamente, la dimensione ecclesiale della Pentecoste. Il dono dello Spirito era così legato non solo a una preparazione individuale, pur necessaria, ma anche a una comunità in preghiera e in devoto ascolto della Scrittura -mediata e interpretata da sapienti predicatori.

L'esaltazione del ruolo della predicazione emerge in maniera trasversale lungo le varie versioni del sermone Repleti sunt omnes. Sorprendentemente, però, la Pentecoste non è utilizzata per enfatizzare la funzione degli apostoli come primi ed esemplari predicatori, come nei sermoni dei secoli precedenti. Gli apostoli sono qui presentati come discepoli che hanno bisogno di venire istruiti e rincuorati dalle parole della Vergine, la quale in un certo senso ha anche il potere - con la sua intercessione- di "provocare" o "accelerare" la discesa dello Spirito. In ultima analisi, questa riscrittura del racconto di Pentecoste ad usum pulpiti esalta e sublima il ruolo della Vergine. Non a caso, Ferrer riutilizza la medesima invenzione narrativa in un sermone in suo onore, quando discutendo delle sette gioie della Madonna si sofferma sulla Pentecoste, ripresentando il medesimo racconto drammatizzato, così da fare di lei la praedicatrix praedicatorum ${ }^{81}$.

\section{BIBLIOGRAFIA CITATA}

FONTI

Bartolomeo da Breganze, Sermones de beata Virgine (1266), ed. Laura Gaffuri, Padova, Editrice Antenore, 1993.

Expositio hymnorum, [Strasburgo, Johann Grüninger, c. 1488]

Fages, Henri (ed.) (1904), Procès de la canonisation de Saint Vincent Ferrer, Parigi, A. Picard.

Ferrer, Vicent, Sermones de tempore, 3 voll., Colonia, Heinrich Quentell, 1485.

Ferrer, Vicent, Sermons, ed. Josep Sanchis Sivera, 2 voll., Barcellona, Barcino, 1932-1934.

\footnotetext{
${ }^{81}$ Il racconto comprende l'attesa degli apostoli lungo la settimana, la spiegazione della Vergine, la preghiera divisa nei tre gruppi e la discesa dello Spirito; Ferrer 1485, vol. III, f. S7r-v ("De nativitate beate Marie virginis vel de septem gaudiis et semptem tristiciis"). Il sermone è attestato solo nell'edizione a stampa e tra gli schemi del 1407-1408; Perarnau 1999d, n. 253. Ferrer rielabora e applica alla Pentecoste la tradizione -particolarmente forte in ambito domenicano- della Vergine quale apostola apostolorum; si veda Delcorno 2018. Ad esempio, nel 1266 il titolo di apostlorum apostola et doctorum magistra e' collegato al versetto di Sapienza 8,4, utilizzato anche da Ferrer, in Bartolomeo da Breganze 1993, p. 412.
} 
Ferrer, Vicent, Sermonario de San Vicente Ferrer del Real ColegioSeminario del Corpus Christi de Valencia, eds. Francisco Gimeno Blay, María Luz Mandingorra Llavata, Valencia, Ajuntament de Valencia, 2002.

Ferrer, Vicent, Sermonario de Perugia (Convento dei Domenicani, ms. 477), eds. Francisco Gimeno Blay, María Luz Mandingorra Llavata, Valencia, Ajuntament de Valencia, 2006.

Ferrer, Vicent, Sermones, Perugia, Convento dei Domenicani, MS 477.

Ferrer, Vicent, Sermones de tempore, Toulouse Bibliothèque municipale MS 345.

Hesbert, René-Jean (ed.) (1963-1979), Corpus antiphonalium Officii, 6 voll., Roma, Herder.

Iacopo da Varazze, Legenda aurea, ed. Giovanni Paolo Maggioni, Impruneta, SISMEL-Edizioni del Galluzzo, 1998.

STUDI

Bériou, Nicole (2018), Religion et communication: un autre regard sur la prédication au Moyen Âge, Ginevra, Droz.

Bouhaïk-Gironès, Marie; Polo de Beaulieu, Marie-Anne (eds.) (2013), Prédication et performance du XII ${ }^{e}$ au XVI ${ }^{e}$ siècle, Parigi, Classiques Garnier.

Boynton, Susan; Reilly, Diane J. (eds.) (2011), The Practice of the Bible in the Middle Ages: Production, Reception, and Performance in Western Christianity, New York, Columbia University Press.

Cabré, Lluís; Renedo Puig, Xavier (1996), "Et postea aplicetur thema". Format in the Preaching of St Vincent Ferrer OP, "Archivum Fratrum Praedicatorum" 66, pp. 245-256.

Callado Estela, Emilio (ed.) (2007), El fuego y la palabra: San Vicente Ferrer en el 550 aniversario de su canonización (Valencia, 26-29 de abril de 2005), Valencia, Generalitat valenciana.

Catalán Casanova, Oriol (2013), La predicació cristiana a la Catalunya baixmedieval: un instrument de transformació cultural, Barcellona, Universitat de Barcelona (Tesi di dottorato), http://www.tdx.cat/ handle/10803/119770 [consultato: 08/03/2018].

Cátedra García, Pedro (1994), Sermón, sociedad y literatura en la Edad Media. San Vicente Ferrer en Castilla (1411-1412): estudio bibliográfico, literario y edición de los textos inéditos, Valladolid, Junta de Castilla y León.

Cooper-Rompato, Christine (2016), Xenoglossia, Multilingualism in Middle English Sermons on Pentecost, in Classen, Albrecht (ed.), 
Multilingualism in the Middle Ages and Early Modern Age: Communication and Miscommunication in the Premodern World, Berlino, De Gruyter, pp. 233-248.

Delcorno, Carlo (1996), La trasmissione nella predicazione, in Giuseppe Cremascoli e Claudio Leonardi (eds.), La Bibbia nel Medio Evo, Bologna, EDB, pp. 65-86.

Delcorno, Carlo (2006), Vicent Ferrer e l'Osservanza francescana, in Delcorno, Carlo, "Quasi quidam cantus". Studi sulla predicazione medievale, eds. Giuseppe Baffetti, Giorgio Forni, Silvia Serventi, Firenze, Olschki, 2009, pp. 263-290.

Delcorno, Pietro (2011), 'Faré per manera que vàlgue per molts'. I sermoni di Vicent Ferrer sulla parabola di Lazzaro e il ricco epulone, "Erebea" 1, pp. 203-229.

Delcorno, Pietro (2014a), Lazzaro e il ricco epulone: Metamorfosi di una parabola fra Quattro e Cinquecento, Bologna, Il Mulino.

Delcorno, Pietro (2014b), "We Have Made It for Learning": the FifteenthCentury Florentine Religious Play Lazero ricco e Lazero povero as a Sermon in the Form of Theatre, in Muzzarelli, Maria Giuseppina (ed.), From Words to Deeds. The Effectiveness of Late Medieval Preaching, Turnhout, Brepols, pp. 65-97.

Delcorno, Pietro (2017a), In the Mirror of the Prodigal Son: The Pastoral Uses of a Biblical Narrative (c. 1200-1550), Leiden, Brill.

Delcorno, Pietro (2017b), Late Medieval Preaching, in Szarmach, Paul E. (ed.), Oxford Bibliographies in Medieval Studies, Oxford, Oxford University Press. DOI: 10.1093/OBO/9780195396584-0239.

Delcorno, Pietro (2017c), "Et ista sunt scripta Dantis": Predicare la Commedia in Quaresima, in Delcorno, Pietro; Lombardo, Eleonora; Tromboni, Lorenza (eds.), I sermoni quaresimali: digiuno del corpo, banchetto dell'anima, "Memorie Domenicane" 48, pp. 125-143.

Delcorno, Carlo (2018), "A Lei nel principio andiamo". I prothema con Maria, "Revue Mabillon" 29, in corso di stampa.

Delcorno, Pietro (2019), Late Medieval Preaching on Pentecost: the Holy Spirit in Five Model Sermon Collections, in Marin, Olivier; Viallet, Ludovic (eds.) (2019), Pentecôtes médiévales. Fêter la venue de l'Esprit Saint dans l'Église latine (VIII siècle-XVI siècle), Rennes, Presses Universitaires de Rennes, in corso di stampa.

Delmas, Sophie (2019), "Per Spiritus Sancti desideratam influentiam”: les sermons pour Pentecôte au XIII siècle, in Marin, Olivier; Viallet, Ludovic (eds.) (2019), Pentecôtes médiévales. Fêter la venue de l'Esprit Saint dans l'Église latine (VIII siècle-XVI siècle), Rennes, Presses Universitaires de Rennes, in corso di stampa. 
Esponera Cerdán, Alfonso (2007), La Palabra de Dios es fuego que inflama y martillo que ablanda la dura piedra del corazón en el amor. Las Sagradas Escrituras y San Vicente Ferrer, "Escritos del Vedat" 37, pp. 303-324.

Fuster, Joan (1954), Notes per a un estudi de l'oratòria vecentina, "Revista Valenciana de Filología" 4/2-4, pp. 87-185. [Lavoro pubblicato con il titolo, L'oratòria de sant Vicent Ferrer, en: Fuster, Joan (1962), Llengua, literatura, historia, Barcelona, Edicions 62, pp. 23-151].

Gaffuri, Laura (2006), "In partibus illis ultramontanis". La missione subalpina di Vicent Ferrer (1402-1408), in Hodel, Paul-Bernard; Morenzoni, Franco (eds.), Mirificus Praedicator. À l'occasion du sixième centenaire du passage de saint Vincent Ferrer en pays romand, Roma, Istituto Storico Domenicano, pp. 105-120.

Gneuss, Helmut (1968), Hymnar und Hymnen im englischen Mittelalter, Tubinga, Niemayer.

Kienzle, Beverly Mayne (ed.) (2000), The Sermon, Turnhout, Brepols.

Longhi, Roberto (1974), Giudizio sul Duecento e ricerche sul Trecento nell'Italia centrale, Firenze, Sansoni.

Losada, Carolina (2015), Powerful Words: St Vincent Ferrer's Preaching and the Jews Medieval Castile, in Cohen, Thomas; Twomey, Lesley (eds.), Spoken Word and Social Practice. Orality in Europe (14001700), Leiden, Brill, pp. 206-227.

Maldina, Nicolò (2016), Raccogliendo briciole. Una metafora della formazione dantesca tra Convivio e Commedia, "Studi Danteschi" 81, pp. 131-164.

Marin, Olivier; Viallet, Ludovic (eds.) (2019), Pentecôtes médiévales. Fêter la venue de l'Esprit Saint dans l'Église latine (VIII siècle-XVI siécle), Rennes, Presses Universitaires de Rennes, in corso di stampa.

Martínez Romero, Tomàs (2002), Aproximació als sermons de Sant Vicent Ferrer, Valencia, Denes Editorial.

Moss, Ann (1987), Latin Liturgical Hymns and Their Early Printing History, 1470-1520, "Humanistica Lovaniensia" 36, pp. 112-137.

Muessig, Carolyn (ed.) (2002), Preacher, Sermon and Audience in the Middle Ages, Leiden, Brill.

Muessig, Carolyn (2015), Bernardino da Siena and Observant Preaching as a Vehicle for Religious Transformation, in Mixson, James D.; Roest, Bert (eds.), A Companion to Observant Reform in the Later Middle Ages and Beyond (1400-1550), Leiden, Brill, pp. 185-203.

Muzzarelli, Maria Giuseppina (ed.) (2014), From Words to Deeds. The Effectiveness of Late Medieval Preaching, Turnhout, Brepols. 
Perarnau Espelt, Josep (1985), La compilació de sermons de Sant Vicent Ferrer de Barcelona, Biblioteca de Catalunya, MS 477, "Arxiu de textos catalans antics" 4, pp. 213-402.

Perarnau Espelt, Josep (1999a), Cent anys d'estudis dedicats als sermons de Sant Vicent Ferrer, "Arxiu de textos catalans antics" 18, pp. 10-62.

Perarnau Espelt, Josep (1999b), Els manuscits d'esquemes $i$ de notes de sermons de Sant Vincent Ferrer, "Arxiu de textos catalans antics" 18, pp. 157-398.

Perarnau Espelt, Josep (1999c), Sobre el manuscrit de València, Col·legi del Patriarca, amb sermons de sant Vicent Ferrer, "Arxiu de textos catalans antics" 18, pp. 399-453.

Perarnau Espelt, Josep (1999d), Aportació a un inventari de sermons de Sant Vicent Ferrer: temes bíblics, títols i divisions esquemàtiques, "Arxiu de textos catalans antics" 18, pp. 479-811.

Perarnau Espelt, Josep (2011-2013), Vària sobre Sant Vicent Ferrer, “Arxiu de textos catalans antics" 30, pp. 527-561.

Renedo Puig, Xavier (2007), Del quadern al sermó, i del sermó al manuscrit. Unitat $i$ diversitat en els sermons de Sant Vicent Ferrer, in Callado Estela, Emilio (ed.) (2007), El fuego y la palabra: San Vicente Ferrer en el 550 aniversario de su canonización (Valencia, 26-29 de abril de 2005), Valencia, Generalitat Valenciana, pp. 89-110.

Riquer, Martí de (1963/64), Fecha y localización de algunos sermones de San Vicente Ferrer, "Boletín de la Real Academia de Buenas Letras de Barcelona" 30, pp. 151-168.

Rusconi, Roberto (1989), Reportatio, in Rusconi (2016), pp. 33-62.

Rusconi, Roberto (2008), Declinazioni iconograficje della santità: Le rappresentazioni di Vicent Ferrer nel corso del XV secolo, in Rusconi (2016), pp. 335-372.

Rusconi, Roberto (2016), Immagini dei predicatori e della predicazione in Italia alla fine del Medieoveo, Spoleto, Centro Italiano di Studi Sull'Alto Medioevo.

Sánchez Sánchez, Manuel Ambrosio (2000), Vernacular Preaching in Spanish, Portuguese and Catalan, in Kienzle, Beverly Mayne (ed.), The Sermon, Turnhout, Brepols, pp. 759-858.

Sánchez Sánchez, Manuel Ambrosio (2011), Dos décadas de estudio sobre predicación en la España medieval, "Erebea" 1, pp. 3-20.

Santonja Hernández, Pedro (2012), La disputa de Tortosa. Jerónimo de Santa Fe y san Vicente Ferrer, "Helmantica" 63, pp. 133-152.

Thayer, Anne (2014), Medieval Sermon Studies since "The Sermon": A Deepening and Broadening Field, "Medieval Sermon Studies" 58, pp. 10-27. 
Vecchio, Silvana (1997), Les langues de feu: Pentecôte et rhétorique sacrée dans les sermons des XII et XIII ${ }^{e}$ siècles, in Dessì, Rosa Maria; Lauwers, Michel (eds.), La parole du prédicateur ( $V^{e}-X V^{e}$ siècle), Turnhout, Brepols, pp. 255-269.

Vecchio, Silvana (2008), "Dispertitae linguae": Le récit de la Pentecôte entre exégèse et prédication, in Moos, Peter von (ed.), Zwischen Babel und Pfingsten: Sprachdifferenzen und Gesprächsverständigung in der Vormoderne (8.-16. Jahrhundert), Zurigo, Lit Verlag, pp. 237-251.

Vidal Doval, Rosa (2010), Predicación y persuasión: Vicente Ferrer en Castilla, 1411-1412, "Revista de poética medieval" 24, pp. 225-243.

Walsh, Peter G.; Husch, Christopher (eds.) (2012), One Hundred Latin Hymns: Ambrose to Aquinas, Cambridge, MA, Harvard University Press.

Wenzel, Siegfried (2012), The Use of the Bible in Preaching, in Marsden, Richard; Matter, E. Ann (eds.), The New Cambridge History of the Bible from 600 to 1450, Cambridge, Cambridge University Press, pp. 680-692.

Fecha de recepción del artículo: abril 2018

Fecha de aceptación y versión final: noviembre 2018 